\title{
INTRA-CAVITY PULSE COMPRESSION IN PASSIVELY MODE-LOCKED Nd-GLASS LASERS
}

\author{
J. SCHMIDT, F. REIL and A. PENZKOFER \\ Naturwissenschaftliche Fakultät II - Physik, Universität Regensburg, 8400 Regensburg, Fed. Rep. Germany
}

Received 19 February 1986; revised manuscript received 25 April 1986

\begin{abstract}
The spectral gain width of a passively mode-locked Nd-phosphate glass laser is reduced with a birefringent filter, and towards the end of the pulse train self-phase modulated pulses are temporally compressed by the restricted gain width. Short pulses upon broad pedestals are formed. The wings are reduced by passing the pulses through an external saturable absorber. Under favorable conditions the pulse durations are shortened from about 7 ps to about $1.5 \mathrm{ps}$.
\end{abstract}

\section{Introduction}

In passively mode-locked Nd-phosphate glass lasers nearly bandwidth limited pulses of 5 to 7 ps duration are generated in the rising part of the pulse train [14]. The spectral width of the pulses $\left(\Delta \widetilde{\nu}_{\mathrm{L}} \simeq 0.5 / c \Delta t_{\mathrm{L}}\right.$ $\simeq 3 \mathrm{~cm}^{-1}$ ) is small compared to the inhomogeneously broadened spectral fluorescence width of the active medium $\left(\Delta \widetilde{\nu}_{\mathrm{F}} \simeq 200 \mathrm{~cm}^{-1}\right)$. Towards and beyond the pulse train maximum the pulses become self-phase modulated [5-15]. The spectral broadening by selfphase modulation increases with the number of roundtrips in the oscillator. The spectral gain profile of the active medium deforms the temporal shape of selfphase modulated pulses [16-21] .

At moderate pulse intensities the spectral broadening by self-phase modulation in the oscillator remains small compared to the gain width and the temporal pulse deformation is small even towards the end of the pulse train [22]. Under conditions of very intense picosecond pulse generation (high saturable absorber concentration, high mirror reflectivity, beam focusing by lenses or mirrors) the spectral broadening may extend beyond the bandwidth of the active medium and strong temporal pulse deformation may occur. The depletion of inversion at the central laser frequency (spectral hole burning in inhomogeneous gain profile) $[17,22-25]$ enhances the pulse disintegration and subpicosecond pulse break-up is observed [5-15] within the pulse envelope (see curve 3 in fig. $2 b$ of ref. [17]).

In this paper intracavity pulse compression of moderately self-phase modulated pulses is achieved by spectral narrowing the gain profile with a birefringent filter. A central spike upon a broad pedestal is formed. The signal height of the wings is reduced by passing the pulses through an external saturable absorber.

\section{Principle}

A laser pulse of electrical field strength $E=E_{0}(t)$ $\times \exp \left[\mathrm{i}\left(\omega_{0} t-k_{0} z-\phi\right)\right]=E_{0}(t) \exp \left[\mathrm{i}\left(\omega_{0} t^{\prime}-\phi\right)\right]$ becomes phase modulated when passing through a nonlinear medium of length $l\left(t^{\prime}=t-n_{0} z / c, k_{0}\right.$ $\left.=\omega_{0} n_{0} / c\right)$. The phase change is $\phi=\left(\omega_{0} / c\right) \gamma \int_{0}^{l} I(t, z)$ $X \mathrm{~d} z$ where $\gamma=n_{2} /\left(n_{0} c \epsilon_{0}\right)$ is the nonlinear refractive index constant $\left(n=n_{0}+\gamma I=n_{0}+n_{2} E_{0}^{2} / 2\right)[26]$. The frequency change is given by $\omega-\omega_{0}=-\partial \phi / \partial t^{\prime}$ $=-\left(\omega_{0} / c\right) \gamma \int_{0}^{l}\left(\partial I / \partial t^{\prime}\right) \mathrm{d} z$.

The situation is depicted in fig. 1 . The solid curve in part (a) shows an asymmetric pulse shape with steeper rising than trailing edge. Such pulse shapes are formed in passively mode-locked Nd-glass lasers by the action of the saturable absorber [27] .

The frequency chirp of the pulse is illustrated in part (b). The spectral gain profile $g(\omega)$ (solid curve) and the modulated pulse spectrum $I(\omega)$ (dashed 

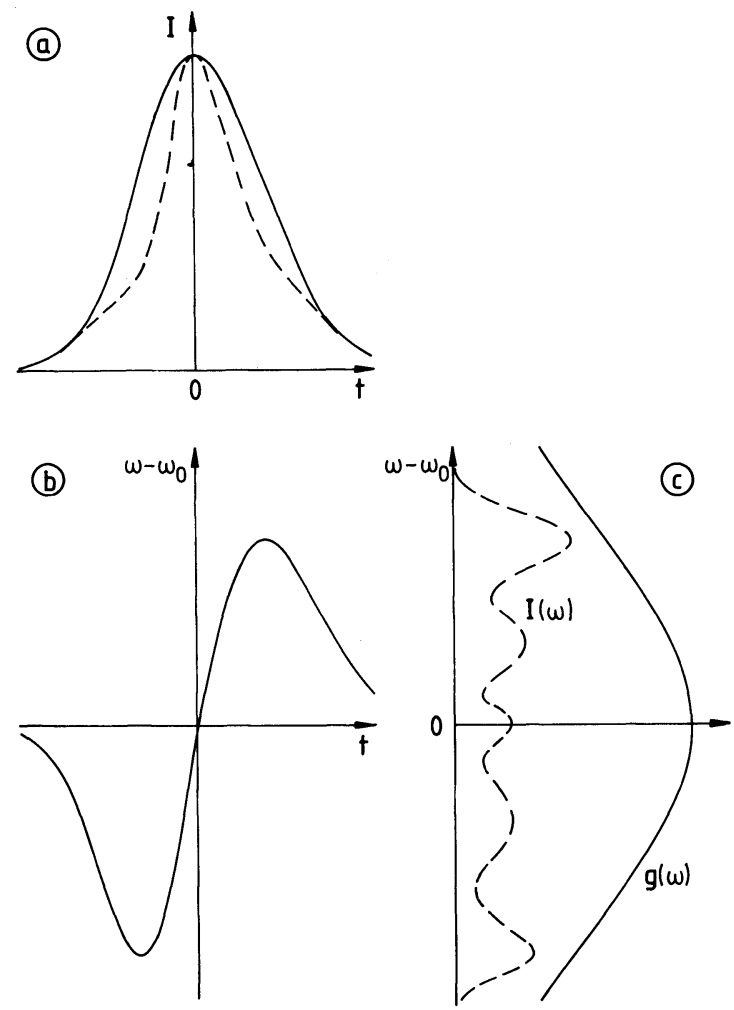

Fig. 1. Description of temporal pulse shortening of self-phase modulated pulses by passage through active medium of finite spectral gain width. (a) Solid curve, initial temporal pulse shape. Dashed curve, final temporal pulse shape after one round-trip. (b) Frequency chirp. (c) Spectral gain profile $g(\omega)$ and spectral intensity distribution $I(\omega)$.

curve) are depicted in picture (c) $[27,28]$. The gain profile changes the temporal pulse shape and the dashed curve of part (a) is the result.

\section{Experimental}

The experimental setup is shown in fig. 2. The laser oscillator consists of two mirrors $\mathrm{M} 1$ and $\mathrm{M} 2$, a contacted dye cell DC1, a Brewster angled dye cell DC2, a Nd-phosphate laser rod LR and a birefringent filter BRF. A single pulse is selected from the pulse train by a Kerr cell shutter which is operated by a laser triggered spark gap. Pulse selection beyond the pulse train maximum is achieved by insertion of a delay cable CA2 between spark gap and Kerr cell. In some of the

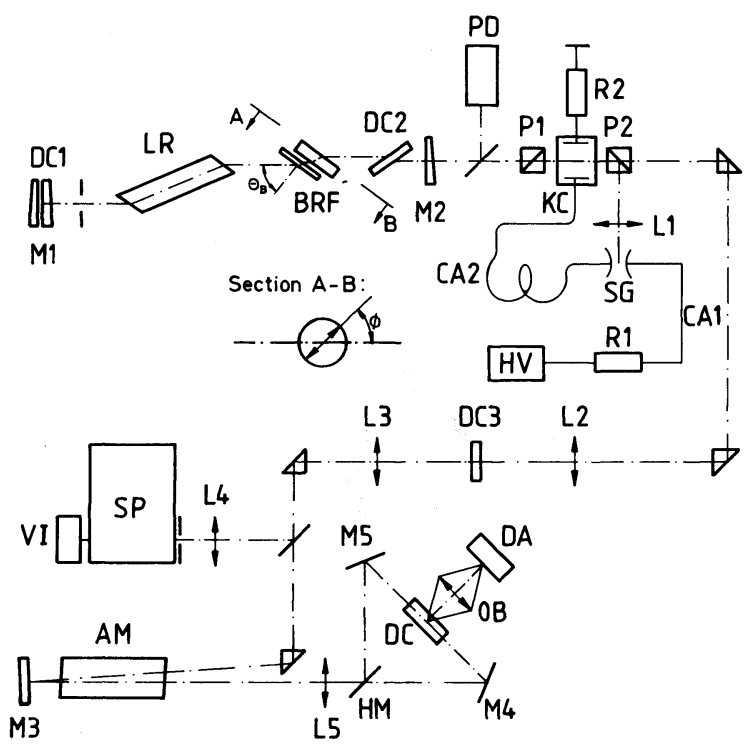

Fig. 2. Experimental arrangement. M1-M5, laser mirrors $[R(\mathrm{M} 2)=0.7] . \mathrm{DC} 1$, contacted dye cell (dye Kodak No. 9860). DC2, DC3, dye cells with Kodak dye No. 5. LR, Ndphosphate glass rod (Schott type LG 703). BRF, birefringent filter with one or two plates, thicknesses $t_{1}=5 \mathrm{~mm}$ (BRF1) and $t_{2}=10 \mathrm{~mm}$ (BRF2), refractive indices at $\lambda=1.053 \mu \mathrm{m}$, $n_{0}=1.534292$ and $n_{\mathrm{e}}=1.543039$. PD, photodetector. P1, P2, Glan polarizers. KC, Kerr cell. L1-L5, lenses. SG, spark gap. $\mathrm{HV}$, high voltage power supply. $\mathrm{R} 1,1 \mathrm{G} \Omega$ resistor. CA1, pulse forming cable. CA2, pulse delay cable. R2, matching terminal resistor. AM, Nd-phosphate glass amplifier (type Hoya LHG7). HM, 50 percent mirror. DC, two-photon absorbing dye cell. OB, objective. DA, diode array system. SP, $60 \mathrm{~cm}$ grating spectrometer (grating 1200 lines/mm). VI, vidicon system.

experiments the selected single pulse is passed through a saturable absorber cell DC3 before entering the Ndphosphate glass amplifier. The spectrum of the selected pulses is analysed with a grating spectrometer. The duration of the amplified pulses is measured by a two-photon fluorescence arrangement (dye 2.5 $\times 10^{-3}$ molar rhodamine $6 \mathrm{G}$ in ethanol).

A one-plate or two-plate birefringent filter between the laser rod and the saturable absorber cell (all elements under Brewster angle) is used for spectral narrowing the gain profile of the active medium. In figs. $3 \mathrm{a}$ and $\mathrm{b}$ the spectral transmission $T_{\mathrm{B}}$ is shown for the one-plate and two-plate birefringent filter, respectively [30-32]. The curves give the transmission for one round-trip starting between laser rod and birefringent 


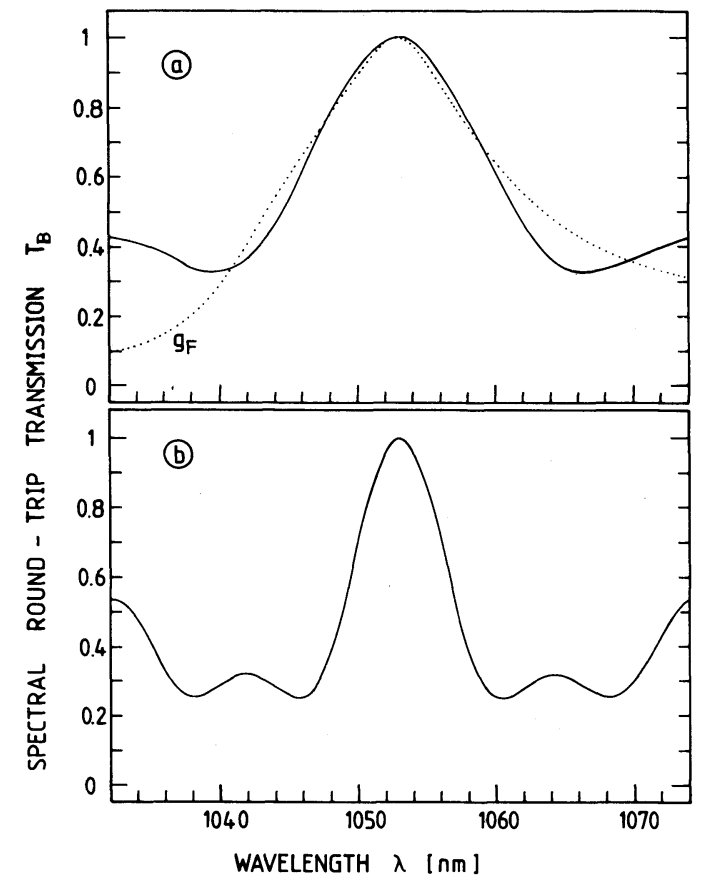

Fig. 3. Spectral transmission per round-trip through oscillator of fig. 2 with single plate filter (a) and two-plate filter (b). Orientation of birefringent filter $\theta_{B}=56.9^{\circ}, \phi=49.46^{\circ}$. The curves are calculated using the theory of ref. [32] . In (a) spectral shape $g_{\mathrm{F}}$ of fluorescence emission of Nd-phosphate glass rod is included (dotted curve).

filter with parallel polarized light. In fig. 3a the spectral shape $g_{\mathrm{F}}(\omega)$ of the flash-lamp excited fluorescence emission of the Nd-phosphate glass rod is included (dotted curve). This fluorescence profile determines the gain profile $g_{\mathrm{G}}(\omega)$ of the active medium [4]. The effective gain profile of the oscillator is given by $g(\omega)$ $=g_{\mathrm{G}}(\omega) T_{\mathrm{B}}(\omega)$. The inhomogeneous gain profile $g_{\mathrm{G}}(\omega)$ changes along the pulse train due to inversion depletion by the laser transition [17].

\section{Results}

In figs. 4 to 6 some results of the pulse development are presented for the resonator with a two-plate birefringent filter and two saturable absorbers $\left(T_{\mathrm{DC} 1}\right.$ $=0.85$, single pass transmission, dye Kodak No. 9860; $T_{\text {DC2 }}=0.85$, dye Kodak No. 5 ).

In fig. 4a a typical pulse train is shown. For the in-

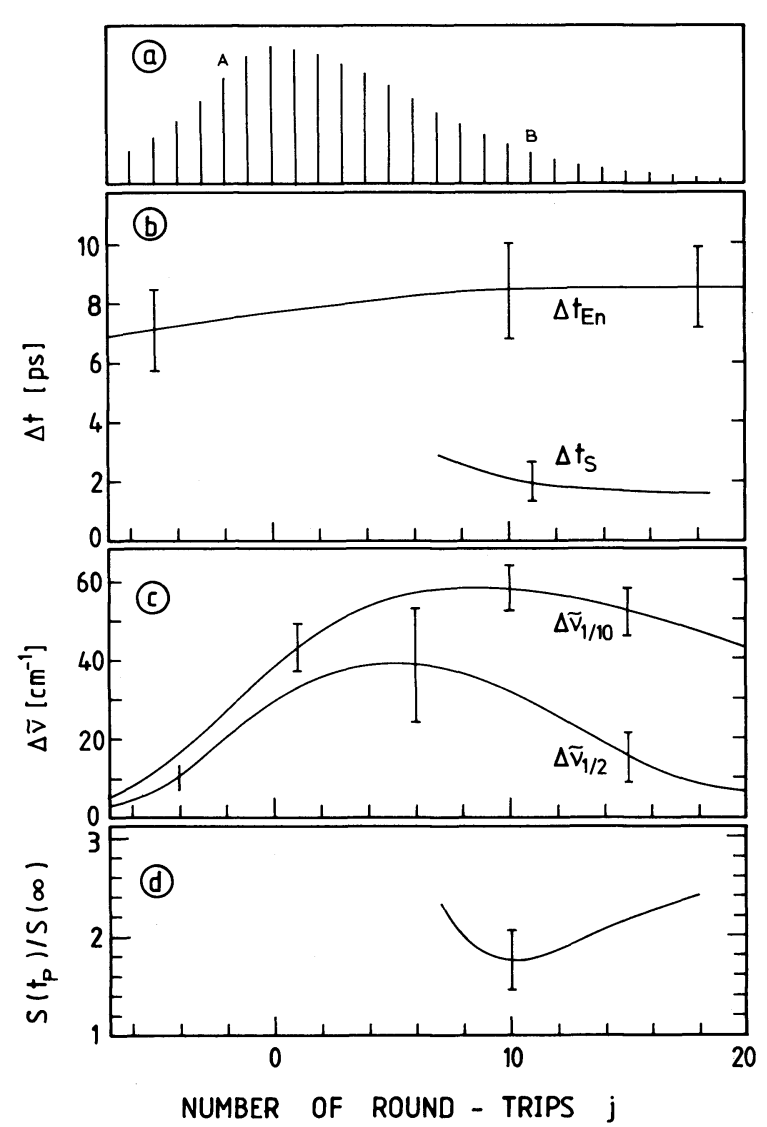

Fig. 4. Pulse development in oscillator with two-plate birefringent filter and two saturable absorbers in DC1 (Kodak No. 9860, single pass transmission $T_{0}=0.85$ ) and DC2 (Kodak No. $5, T_{0}=0.85$ ). (a) Typical pulse train. (b) Envelope pulse duration- $\Delta t_{\text {En }}(\mathrm{fwhm})$ and duration of central spike $\Delta t_{\mathrm{S}}$ (fwhm). (c) Spectral widths at half height $\Delta \widetilde{\nu}_{1 / 2}$ (fwhm) and at one tenth height $\Delta \widetilde{\nu}_{1 / 10}$. (d) Normalized height $S\left(t_{\mathrm{p}}\right) / S(\infty)$ of side maximum in two-photon fluorescence trace. The solid curves in $(b-d)$ are least-square fits to the actual experimental data. The error bars are typical single-shot standard deviations for different round-trip positions $j$.

dicated positions $\mathrm{A}$ and $\mathrm{B}$, the two-photon fluorescence traces and the pulse spectra are shown in fig. 5a and fig. 6 , respectively. The decorrelated pulse shapes are depicted in fig. $5 \mathrm{~b}$. The decorrelation procedure is described in ref. [33]. The pulse at position A near the pulse train maximum has the shape of a single pulse of 6.2 ps duration. Its spectral width is $\Delta \widetilde{\nu}_{1 / 2}$ $\simeq 17 \mathrm{~cm}^{-1}$ (fig. 6a). The pulse is already spectrally broadened by self-phase modulation $(\Delta \nu \Delta t \simeq 3.2)$. The pulse at position $B$ is composed of a short spike of 

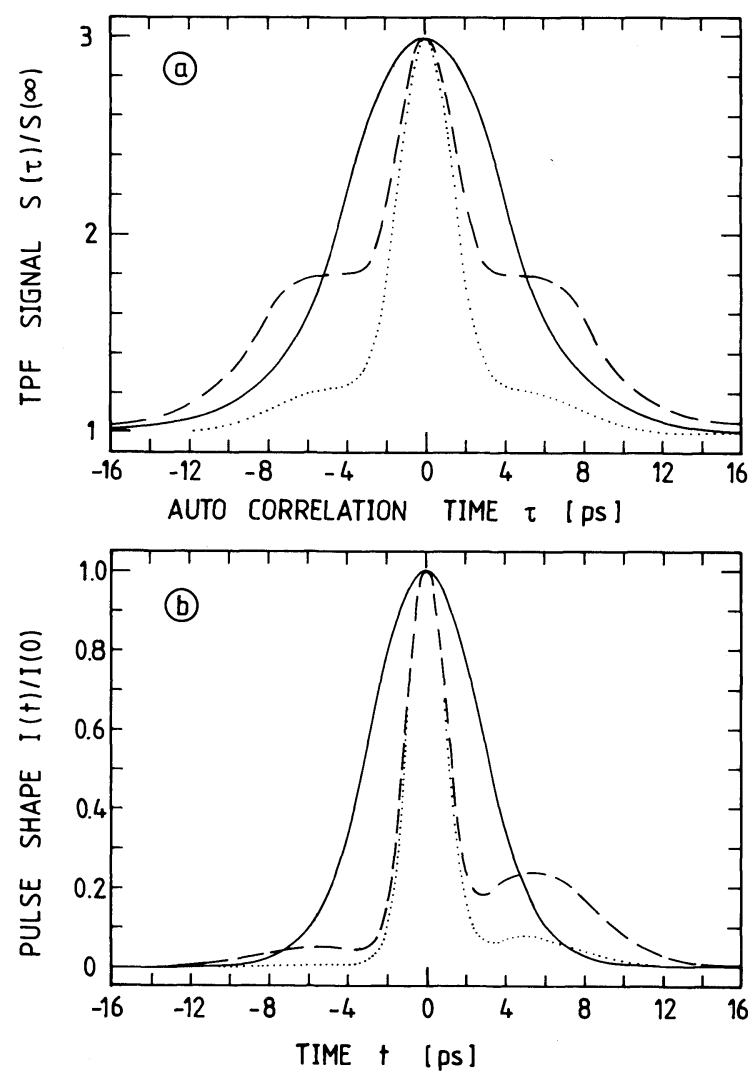

Fig. 5. Two-photon fluorescence traces (a) and corresponding decorrelated temporal pulse shapes (b). Oscillator with twoplate birefringent filter and two saturated absorbers. Solid curves, pulse at position A of fig. 4. Dashed curves, pulse at position B of fig. 4. Dotted curve, pulse from position B of fig. 4 passed through external absorber in DC3 (Kodak No. 5, $T_{0}=0.03$ ).

width $\Delta t_{\mathrm{S}} \simeq 2.3 \mathrm{ps}$ and a broad pulse of width $\Delta t_{\mathrm{L}}$ $\simeq 7.2$ ps separated from the short pulse by $t_{\mathrm{p}} \simeq 5.5 \mathrm{ps}$. A leading pulse (at $\tau=-t_{\mathrm{p}}$ ) is not observed (leading wing of fig. 1a is absorbed by saturable dyes). The corresponding pulse spectrum is modulated (fig. 6b). It peaks at the short wavelength side and has a halfwidth of $\Delta \widetilde{\nu}_{1 / 2} \simeq 46 \mathrm{~cm}^{-1}$. The spectral extension at one tenth of the peak height $\Delta \widetilde{\nu}_{1 / 10}$ is approximately equal to the effective gain width of the active medium-birefringent filter combination.

The complete temporal and spectral pulse developments along the pulse. train are depicted in figs. $4 \mathrm{~b}$ and $4 c$, respectively. In fig. $4 \mathrm{~b}$ the envelope pulse dur-

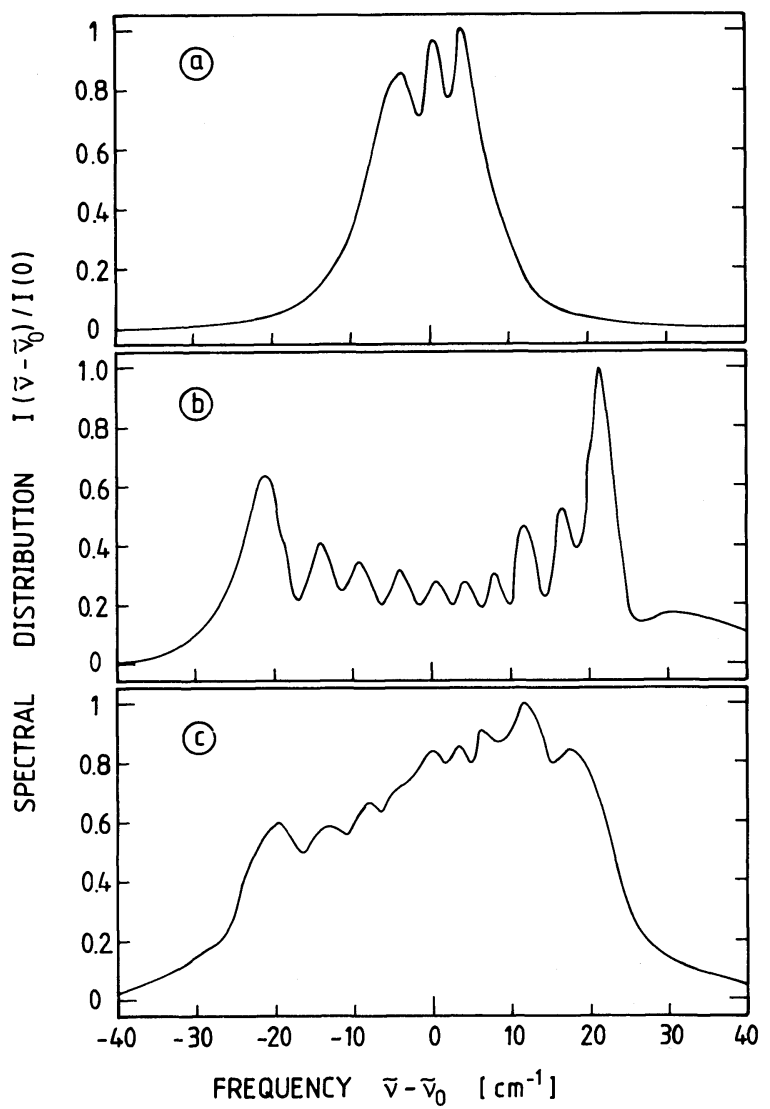

Fig. 6. Spectral shapes of pulses depicted in fig. 5. (a) At position A of fig. 4. (b) At position B of fig. 4. (c) After passage through external absorber.

ation $\Delta t_{\mathrm{En}}(\mathrm{fwhm})$ and - in the region of existence the central spike duration $\Delta t_{\mathrm{S}}(\mathrm{fwhm})$ are shown. Without the two-plate birefringent filter single picosecond pulses of 5 to 6 ps duration would be generated. The spectral widths $\Delta \widetilde{\nu}_{1 / 2}($ fwhm $)$ and $\Delta \widetilde{\nu}_{1 / 10}$ (full width at one tenth height) are depicted in fig. $4 \mathrm{c}$. The spectral half-widths fluctuate strongly in the trailing part of the pulse train. Broad half-widths include the short and long wavelengths side peak of the modulated spectrum. Small half-widths extend only over the short wavelength peak (see fig. 1c). This situation dominates towards the end of the pulse train. In fig. $4 \mathrm{~d}$ the contrast ratio $S\left(t_{\mathrm{p}}\right) / S(\infty)$ of the fluorescence traces is depicted for the region where short pulses upon pedestals are formed $\left(\tau=t_{\mathrm{p}}\right.$ is position of side pulse maximum). The contrast ratio at zero delay is 
Table 1

Laser data at position of shortest central spike formation. Single shot standard deviations are indicated

System configuration:

Plates

$T_{\text {DC1 }}$ (No. 9860)

$T_{\mathrm{DC} 2}$ (No. 5)

$T_{\text {DC3 }}$ (No. 5)
BRF1

\begin{tabular}{lll}
\hline 0.83 & 0.85 & 0.85 \\
1 & 0.85 & 0.85 \\
1 & 1 & 0.03
\end{tabular}

$\mathrm{BRF} 1+\mathrm{BRF} 2$

\begin{tabular}{lll}
\hline 0.83 & 0.85 & 0.85 \\
1 & 0.85 & 0.85 \\
1 & 1 & 0.03
\end{tabular}

Results:

\begin{tabular}{|c|c|c|c|c|c|c|c|c|c|c|}
\hline$\Delta t_{\mathrm{En}}[\mathrm{ps}]$ & $7 \pm 1$ & $7 \pm 1$ & & & $8.5 \pm$ & \pm 1.5 & 8.5 & \pm 1.5 & & \\
\hline$\Delta t_{\mathrm{S}}[\mathrm{ps}]$ & $2.0 \pm 0.6$ & $1.7 \pm 0.4$ & 1.5 & $\pm \quad 0.4$ & $2.5 \pm$ & \pm 0.5 & 2 & \pm 0.5 & 2 & \pm 0.5 \\
\hline$\Delta t_{\mathrm{L}}[\mathrm{ps}]$ & $3.5 \pm 0.5$ & $3 \pm 0.5$ & 3 & \pm 0.5 & $8 \pm$ & \pm 1 & 7 & \pm 1 & 6 & \pm 1 \\
\hline$t_{\mathrm{p}}[\mathrm{ps}]$ & $3.2 \pm 0.5$ & $3.2 \pm 0.5$ & 3.2 & \pm 0.5 & 6 & \pm 1 & 5 & \pm 0.5 & 5 & \pm 0.5 \\
\hline$\Delta \widetilde{\nu}_{1 / 2}\left[\mathrm{~cm}^{-1}\right]$ & $25 \pm 6$ & $30 \pm 10$ & 35 & \pm 9 & 25 & \pm 10 & 25 & \pm 10 & 27 & \pm 12 \\
\hline$\Delta \widetilde{\nu}_{1 / 10}\left[\mathrm{~cm}^{-1}\right]$ & $70 \pm 10$ & \pm 10 & 70 & \pm 10 & 58 & \pm 6 & 58 & \pm 6 & 54 & \pm 6 \\
\hline$S\left(t_{\mathrm{p}}\right) / S(\infty)$ & $2.4 \pm \quad 0.1$ & $2.1 \pm 0.2$ & 1.8 & \pm 0.3 & $2.1 \pm$ & \pm 0.2 & 1.75 & \pm 0.3 & 1.4 & \pm 0.2 \\
\hline$I\left(t_{\mathrm{p}}\right) / I(0)$ & $0.4 \pm 0.1$ & $0.5 \pm 0.1$ & 0.35 & \pm 0.2 & $0.55 \pm$ & \pm 0.2 & 0.3 & \pm 0.1 & 0.15 & \pm 0.1 \\
\hline$I\left(-t_{\mathrm{p}}\right) / I(0)$ & $0.4 \pm \quad 0.1$ & $0.1 \pm 0.03$ & 0.02 & \pm 0.02 & $0.03 \pm$ & \pm 0.03 & 0.03 & \pm 0.03 & 0.02 & \pm 0.02 \\
\hline
\end{tabular}

$S(0) / S(\infty)=3$. The ratio $S\left(t_{\mathrm{p}}\right) / S(\infty)$ is a measure of the side pulse height to the central pulse height. The lower $S\left(t_{\mathrm{p}}\right) / S(\infty)$ the more the central pulse dominates (see fig. 5). The optimum formation of a short central pulse and weak side pulse occurs at about 10 pulses beyond the pulse train maximum.

In the region of central and side pulse formation the side pulse height may be reduced by passage through an external saturable absorber. The two-photon fluorescence trace and the corresponding pulse shape of such an externally shortened pulse are included in figs. 5a and b, respectively (pulse from position B of fig. 4a). The corresponding pulse spectrum is illustrated in fig. 6c. The side pulse height is reduced to about eight percent of the central pulse height. In case of second harmonic generation the side pulse height would reduce to less than one percent of central pulse height (quadratic dependence) and the pulse duration should reduce to $2^{-1 / 2} \Delta t$.

The presented data in figs. 4-6 belong to a oscillator configuration with a two-plate birefringent filter and two saturable absorbers. Other configurations (two-plate birefringent filter with one saturable absorber, one-plate birefringent filter with one or two saturable absorbers) have been analysed and the results are summarized in table 1 . If only one birefringent filter and one saturable absorber is used (cell DC2 filled with solvent 1,2-dichloroethane), a leading side pulse often remains besides the central and trailing pulse (twophoton fluorescence traces with 5 peaks at $\tau= \pm 2 t_{\mathrm{p}}$, $\pm t_{\mathrm{p}}$ and 0 ) [33]. The use of a one-plate birefringent filter together with two dye cells results in the generation of shorter central pulses with somewhat higher trailing side pulses compared to the two-plate filter situation. Together with an external saturable absorber this configuration allows the nearly background free generation of picosecond pulses of $1.5 \pm 0.4$ ps duration.

\section{Conclusions}

In a passively mode-locked Nd-phosphate glass laser containing a birefringent filter pulse durations down to about 1.5 ps could be generated by the action of the finite spectral gain width on self-phase modulated pulse. The extension of the oscillator to an actively and passively mode-locked system by inclusion of an acousto-optic mode-locker $[16,34]$ may reduce the shot to shot fluctuations and improve the intra-cavity pulse compression. The pulse shortening of self-phase modulated pulses by a spectrally limited gain profile 
or transmission profile is an alternative to the dispersive compression of self-phase modulated pulses by prism or grating pairs [35-37].

\section{References}

[1] A. Laubereau and W. Kaiser, Opto-Electronics 6 (1974) 1.

[2] W. Zinth, A. Laubereau and W. Kaiser, Optics Comm. 22 (1977) 161.

[3] G.R. Fleming, I.R. Harrowfield, A.E.W. Knight, J.M. Morris, R.J. Robbins and G.W. Robinson, Optics Comm. 20 (1977) 36.

[4] F. Graf, G. Pleininger and A. Penzkofer, Appl. Phys. B34 (1984) 123.

[5] M.A. Duguay, D.W. Hansen and S.L. Shapiro, IEEE J. Quant. Electron. QE-6 (1970) 725.

[6] D.J. Bradley, G.H.C. New and S.J. Caughey, Phys. Letters 30A (1969) 78.

[7] E.B. Treacy, Appl. Phys. Letters 14 (1969) 112.

[8] R.R. Cubeddu and O. Svelto, IEEE J. Quant. Electron. QE-5 (1969) 495.

[9] O. Svelto, Appl. Phys. Letters 17 (1970) 83.

[10] R.C. Eckardt, C.H. Lee and J.N. Bradford, Appl. Phys. Letters 19 (1971) 420.

[11] R.C. Eckardt, C.H. Lee and J.N. Bradford, OptoElectronics 6 (1974) 67.

[12] A.N. Zherikhin, P.G. Kryukov, E.V. Kurganova, Yu.A. Matveets, S.V. Chekalin, S.A. Churilova and O.B. Shatberashvili, Sov. J. Phys. JETP 39 (1974) 52.

[13] A.N. Zherikhin, P.G. Kryukov, Yu.A. Matveets and S.V. Chekalin, Sov. J. Quant. Electron. 4 (1974) 525.

[14] A.B. Mironov and O.B. Shatberashvili, Sov. J. Quant. Electron. 4 (1974) 805.

[15] R.C. Eckardt, IEEE J. Quant. Electron. QE-10 (1974) 48.

[16] A.J. Duerinckx, H.A. Vanherzeele, J.L. van Eck and A.E. Siegman, IEEE J. Quant. Electron. QE-14 (1978) 983.
[17] A. Penzkofer and F. Graf, Opt. Quant. Electron. 17 (1985) 219.

[18] H. Nathel, D.M. Guthals and J.M. Clark, Technical Digest of CLEO'84 (Anaheim, California, June 1984) p. 182.

[19] P. Sperber and A. Penzkofer, Optics Comm. 54 (1985) 160.

[20] D. von der Linde and A.M. Malvezzi, Appl. Phys. B37 (1985) 1.

[21] T. Tomie, Jap. J. Appl. Phys. 24 (1985) 1008.

[22] A. Penzkofer and N. Weinhardt, IEEE J. Quant. Electron. QE-19 (1983) 567.

[23] V.I. Nikitin, M.S. Soskin and A.I. Khizhuyak, Sov. J. Quant. Electron. 9 (1979) 1314

[24] J.M. Pellegrino, W.M. Yen and M.J. Weber, J. App1. Phys. 51 (1980) 6332.

[25] S.A. Brawer and M.J. Weber, J. Chem. Phys. 75 (1981) 3516.

[26] F. Shimizu, Phys. Rev. Letters 19 (1967) 1097.

[27] W. Leupacher and A. Penzkofer, Appl. Phys. B29 (1982) 263.

[28] O. Svelto, Progress in Optics 12 (1974) 1.

[29] S.A. Akmanov, R.V. Khokhlov and A.P. Sukhorukov, in: Laser Handbook, Vol. 2, eds. F.T. Arrecchi and E.O. Schultz-Dubois (North-Holland, Amsterdam, 1972) p. 1151.

[30] A.L. Bloom, J. Opt. Soc. Am. 64 (1974) 447.

[31] I.J. Hodgkinson and J.I. Vukusic, Appl. Optics 17 (1978) 1944.

[32] D.R. Preuss and J.L. Gole, Appl. Optics 19 (1980) 702.

[33] P. Sperber and A. Penzkofer, Opt. Quant. Electron. 18 (1986), to be published.

[34] L.S. Goldberg and P.E. Schoen, IEEE J. Quant. Electron. QE-20 (1984) 628.

[35] E.B. Treacy, Phys. Lett. A 28 (1968) 34.

[36] A. Laubereau and D. von der Linde, Z. Naturf. 23A (1970) 1626.

[37] B. Nikolaus and D. Grischkowsky, Appl. Phys. Lett. 42 (1983) 1. 